\title{
Necrotic Bullous Erythema Nodosum Leprosum-a Case Report from a Tertiary Care Centre In Eastern India.
}

\author{
Dr Kalidas Rit ${ }^{1}$, Dr Parthasarathi Chakrabarty ${ }^{2}$, Saswati Chattopadhyay ${ }^{3}$, \\ Anita Nandi (Mitra ${ }^{4}$. \\ ${ }^{l}$ Assistant Prof Dept of Microbiology IPGME\&R Kolkata-20 \\ ${ }^{2}$ Demonstator Dept of Microbiology IPGME\&R Kolkata-20 \\ ${ }^{3}$ Demonstrator Dept of Microbiology IPGME\&R Kolkata-20 \\ ${ }^{4}$ Demonstrator Dept of Microbiology. Medical college, Kolkata-73
}

\begin{abstract}
A patient with lepromatous leprosy while on multidrug therapy (MDT) presented with crops of brightly erythematous tender nodules on both upper and lower extremities along with bullous necrotic lesion over face and trunk of one week duration. The lesions were associated with high grade fever. Bilateral ulnar, radial cutaneous and lateral popliteal nerves were thickened. Slit skin smear showed a bacterial index (BI) of $4+$ with morphological index $40 \%$ and histopathology was consistent with type II reaction. Systemic naproxen, corticosteroid and clofazimine were administered and the patient became symptoms free after a week. On $21^{\text {st }}$ hospitalisation day the patient was discharged and she remained symptom free.
\end{abstract}

Key Words: lepromatous leprosy, multidrug therapy, bullous necrotic lesion

\section{Introduction}

Erythema nodosum leprosum (ENL) is a common manifestation of type II lepra reaction characterised by the appearance of erythematous, tender, nodule or plaques with bilateral symmetrical distribution. ENL occurs in patients with lepromatous leprosy (LL) or occasionally with borderline lepromatous leprosy receiving multidrug therapy ${ }^{[1]}$ but may be present in untreated cases. Various precipitating factors include surgical operation, pregnancy, parturition, lactation, menstruation, intercurrent infection, stress and sometimes therapy ${ }^{[2]}$ Vesicular or bullous necrotic lesion also been reported rarely in severe type II reaction and from India there are only few published reports about it. ${ }^{[3,4]}$ Awareness among clinician has to be increased regarding this entity.

\section{Case report}

A 46 years old female patient presented in Dermatology OPD with crops of brightly erythematous tender nodule of both upper and lower extremities along with bullous necrotic lesion over face (Figure 1) and trunk of one week duration. As per history given by the patient she was on WHO-MB-MDT (World Health Organisation-Multibacillary-Multidrug Resistant Tuberculosis) for previous five months because of lepromatous leprosy. There was no history suggestive of exfoliative dermatitis, epitaxis, iridocyclitis, neuritis, lymphadenopathy or hepatitis. Bilateral thickened and nontender ulnar nerve, radial cutaneous nerve and lateral popliteal nerve were noted. Glove and stocking type of anaesthesia with atrophy of both thenar and hypothenar eminences were found. Madarosis was present along with thickened ear lobules. The patient also had high intermittent fever associated with nausea, loss of appetite, bone pain, myalgia, arthalgia and pedal oedema. Biochemical tests including complete blood count, erythrocytic sedimentation rate (ESR), liver and renal function tests, immune markers and G6PD were done. All test results were within normal range except there was raised ESR $(52 \mathrm{~mm})$ and presence of leucocytosis. PAS stained smear of biopsy material of skin lesion showed diffuse polymorphoneuclear infiltrate along with a few foamy histiocyte and acid fast bacilli. A slit skin smear from six different sites were read as $4+$ on the bacteriological index (BI) scale and have a morphological index (MI) of 40\%. ${ }^{[5]}$ [Figure-2] The patient was admitted and started on prednisolone $60 \mathrm{mg}$ daily, clofazimine $100 \mathrm{mg}$ eight hourly and naproxen $500 \mathrm{mg}$ daily but continued to have new lesions on thigh, trunk and upper arm. On the fifth day onwards her fever subsided and no new lesions developed. She became asymptomatic after a week and prednisolone was gradually reduced to $20 \mathrm{mg}$ daily. Finally on $21^{\text {st }}$ day of hospitalisation the patient was discharged and she remained symptom free. 


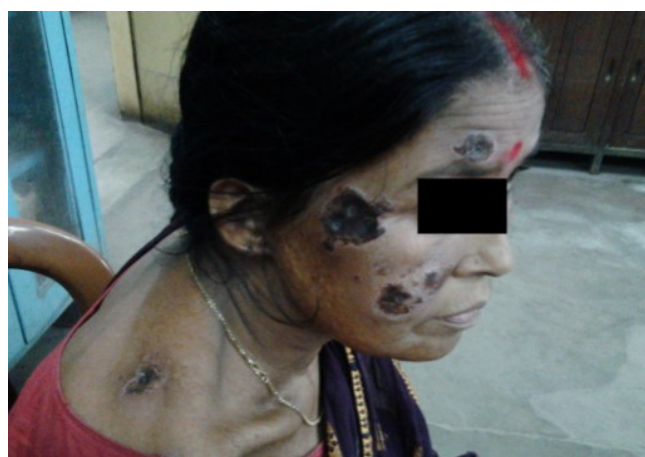

Figure - 1: Bullous necrotic lesion of ENL

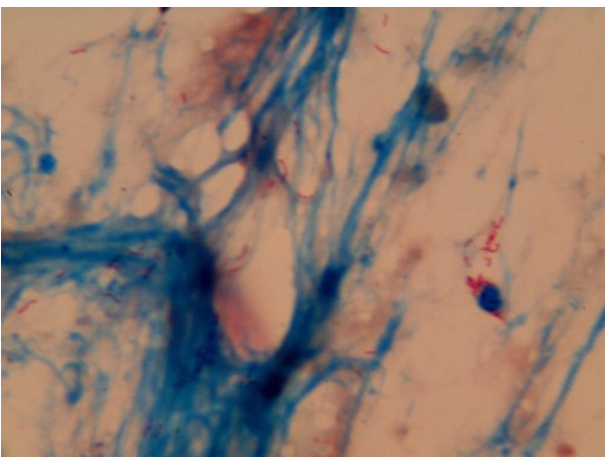

Figure -2: Slit skin smear showing plenty of acid-fast bacilli

\section{Discussion}

Though pustular, ulcerated, erythema multiforme lesions have been reported in lepromatous leprosy, necrotic bullous lesions are infrequent. Generalized bullous eruptions during treatment with rifampicin and dapsone have been reported in the past. Dharmendra and Ramu have described rare incidence of bullous type lepra lesions like the presentation in our case ${ }^{[6]}$ Usually such a bullous lesion in LL indicates acute exacerbation reaction which is rare ${ }^{[3]}$ In our case the bullae appear suddenly without being preceded by any inflammation on the skin. Here the onset was sudden not affecting the mucosa and Nikolsky sign was negative. Practitioners should be alerted to this rare presentation of leprosy. It needs to be differentiated from other common causes of blistering diseases like pemphigus, bullous pemphigoid, erythema multiforme, dermatitis herpetiformis, and bullous drug eruption.

In this case the use of systemic naproxen, prednisolone, and clofazimine were able to control the manifestations of bullous ENL. Naproxen is one of the cheapest NSAID useful in ENL to control moderate degree of pain and inflammation. ${ }^{[7]}$ Controlled use of systemic corticosteroid like prednisolone rapidly control the manifestations of ENL and it is particularly indicated when ENL is associated with neuropathy like that of our case ${ }^{[8]}$ But long term use of systemic high dose of corticosteroid is associated with adverse effects and it should be tapered off gradually to avoid further exacerbation. Clofazimine is useful to reduce the dose and duration of therapy with prednisolone and helpful in weaning patients off corticosteroid. Though variously described as drug of choice in ENL therapy use of thalidomide in controlling the disease manifestation is not be encouraged as per WHO guidelines. ${ }^{[9]}$ Thalidomide is a rapidly acting steroid sparing drug but it has its shortcoming and has to be administered under strict supervision. It is contraindicated in female patients planning a family because of its teratogenic potential. In addition, in many leprosy endemic countries it is either banned or not easily available.

\section{References}

[1]. Pflatzgraff RE, Bryceson A. Clinical leprosy. In: Hastings RC, Opromolla DVA, editors. Leprosy. London: Churchill Livingstone, 1985:165-171.

[2]. Browne S G, Erythema nodosum in leprosy J Chronic Dis 1963; 16:23-30.

[3]. Dogra S, Kaur I, Kumar B. Bullous reaction in leprosy; a rare phenomenon. Int J lepr, 2002; 70: 206-207.

[4]. Davis SV, Shenoi SD, Balachandran C, Pai SB. A fatal case of erythema nectroticans. Indian J Lepr 2002; 74: 145-9. PubMed

[5]. Ridley Ds, Jopling. A classification of leprosy for research purposes. Lepr Rev 1962; 33:119-128.

[6]. Ramu, G. And Dharmendra. Acute exacerbations (reactions) in leprosy. Dharmendra editor. Leprosy, Vol. I, Bombay: Kothari Medical Publishers,1979. 116-123.

[7]. Lehman CW. Control of erythema nodosum with naproxen. Cutis 1980; 26 :66-67.

[8]. Levis WR. Treatment of leprosy in United States. Bull N Acad Med. 1984; 60: 696-711.

[9]. Panikar V. The return of thalidomide: new uses and renewed concerns. Lepr Rev, 2003; 74: 286-287. 